\title{
Political Uncertainty Moderates Neural Evaluation of Incongruent Policy Positions
}

\author{
Ingrid J. Haas \\ Department of Political Science \\ Center for Brain, Biology, and Behavior \\ University of Nebraska-Lincoln \\ Melissa N. Baker \\ Department of Political Science \\ University of California-Merced \\ Frank J. Gonzalez \\ School of Government and Public Policy \\ University of Arizona
}

Final manuscript accepted for publication (2020-12-29).

Please cite as: Haas, I. J., Baker, M. N., \& Gonzalez, F. J. (in press). Political uncertainty moderates neural evaluation of incongruent policy positions. Philosophical Transactions of the Royal Society B. doi: 10.1098/rstb.2020.0138

Word Count: 5276

\author{
Corresponding Author: \\ Ingrid J. Haas \\ Department of Political Science \\ University of Nebraska-Lincoln \\ 531 Oldfather Hall \\ Lincoln, Nebraska 68588 \\ Email: ihaas2@unl.edu
}




\begin{abstract}
Uncertainty has been shown to impact political evaluation, yet the exact mechanisms by which uncertainty affects the minds of citizens remain unclear. This experiment examines the neural underpinnings of uncertainty in political evaluation using functional MRI (fMRI). During fMRI, participants completed an experimental task where they evaluated policy positions attributed to hypothetical political candidates. Policy positions were either congruent or incongruent with candidates' political party affiliation and presented with varying levels of certainty. Neural activity was modeled as a function of uncertainty and incongruence. Analyses suggest that neural activity in brain regions previously implicated in affective and evaluative processing (anterior cingulate cortex, insular cortex) differed as a function of the interaction between uncertainty and incongruence, such that activation in these areas was greatest when information was both certain and incongruent and uncertainty influenced processing differently as a function of the valence of the attached information. These findings suggest that individuals are attuned to uncertainty in the stated issue positions of politicians, and that the neural processing of this uncertainty is dependent on congruence of these positions with expectations based on political party identification. Implications for the study of emotion and politics and political cognition are discussed.
\end{abstract}

Keywords: evaluation, uncertainty, incongruence, fMRI, political cognition, political neuroscience 


\section{Political Uncertainty Moderates Neural Evaluation of Incongruent Policy Positions}

Uncertainty is an inherent part of the political process, given that political issues are ever increasing in complexity and citizens often lack the motivation or ability to fully understand the details. Political scientists have long acknowledged uncertainty as part of the political process (1), and shown, for example, that people are less likely to use information to make decisions about political candidates when that information is subjectively uncertain (2) and that uncertainty can be associated with ambivalence and more negative evaluations of candidates (3). Politicians may project uncertainty about positions they hold on particular issues intentionally or unintentionally. Some empirical work supports the popular assumption that "flip-flopping," equivocating, or being vague has negative consequences for politicians because it portrays them as indecisive $(4,5)$, whereas other work says it is inconsequential given partisan motivations $(6,7)$ or that this indecision can even be viewed positively in the minds of some voters (8). However, this work does not identify the effects of uncertainty, per se, and has revealed somewhat inconsistent findings.

Other political science work has examined the impact of emotions that are likely related to uncertainty, such as anxiety, suggesting that anxiety is likely to lead to a more open-minded cognitive style, whereby people consider new information and are more willing to engage in compromise (9-11). Indeed, past work has shown that uncertainty can lead to increased political tolerance and willingness to compromise, but only under certain conditions $(12,13)$. Uncertainty is more likely to lead to positive outcomes in neutral or positive contexts, but more likely to produce the opposite when paired with negative affect or threat. What remains underexplored at this point is understanding why uncertainty has these effects on political attitudes and beliefs, and how the neural response to uncertainty may change as a function of affective context.

While some emotions or affective states tend to be clearly defined (e.g., fear or threat), uncertainty is a more diffuse affective state. Threat signals the direct potential for harm, whereas uncertainty is simply a signal that we lack information and requires context for 
interpretation. Responses to uncertainty are likely to differ based on what the uncertainty is attached to. In other words, we argue that effects of uncertainty are likely to be contextdependent $(12,13)$. From this perspective, it is important to examine how uncertainty affects political cognition differently when attached to different types of information.

Research in political neuroscience has begun to examine processes involved in political evaluation, such as political candidate perception and evaluation, but has not directly examined how uncertainty influences these processes. Much of this work has presented participants with names or faces of political candidates, and found activation in many of the same brain regions involved in evaluative processing more generally, including the amygdala, insula, anterior cingulate, and orbitofrontal cortex (see e.g., 14, 15, 16). For example, fMRI studies have shown activation in anterior cingulate cortex (ACC), insula, and dorsolateral prefrontal cortex (dIPFC) when people are evaluating disliked or opposition political candidates $(17,18)$. However, other fMRI work has shown that some of these regions also respond to favored candidates (19), suggesting that it may be premature to explain these effects purely on the basis of positive versus negative valence. Many of these brain regions appear to serve multiple functions, and there is ongoing debate about what the exact nature of brain functions in these regions might be. For example, the ACC has been implicated in cognitive control, conflict monitoring, and exploring alternative courses of action (20-22) and is sensitive to uncertainty (23-25). Insula responds to motivationally relevant information or salience and may aid in the process of integrating cognitive with emotional information $(26,27)$ and has also been shown to be sensitive to uncertainty $(28,29)$, although much of this work has been conducted in the context of decision making rather than politics. There is also some evidence that activation in these regions—insula and ACC—may be linked (30). In prior work, we have demonstrated using functional MRI (fMRI) that insula and ACC respond to incongruent (versus congruent) policy statements from hypothetical political candidates, and that this effect was more likely to occur when evaluating own party (versus out party) candidates and for participants who identified as 
politically liberal versus conservative (31). However, we have yet to investigate the role of uncertainty in candidates' stated issue positions, which is of particular importance given the prevalence of uncertainty in contemporary electoral politics. In particular, we are interested here in whether people respond differently to political uncertainty as a function of affective context.

\section{Overview of Present Work}

In the present work, we used functional MRI (fMRI) to examine the impact of both uncertainty and incongruent information on political evaluation. Although work in political science has typically examined unidirectional effects of uncertainty on political evaluation, we expect the effects of uncertainty to depend on the context of evaluation-specifically, whether the information given with respect to a candidate is congruent with the candidate's party identification. We test this by leveraging instances of issue positions conflicting with candidates' party identification as an opportunity to study when context determines the effects of uncertainty on political evaluation. We focus our inquiry on brain regions previously implicated in evaluative processing, including the insula and anterior cingulate. Given past research in cognitive neuroscience, it is unclear whether some areas in the brain are just sensitive to uncertainty regardless of context, or if uncertainty is having effects on processing more indirectly. We think it is unlikely that there is an "uncertainty area" of the brain, but rather uncertainty may change the motivational relevance of different types of information. Past research might suggest that either uncertainty or incongruence would activate regions such as insula or ACC, but we expect to find evidence for an interaction between uncertainty and incongruence.

\section{Method}

\section{Participants}

The analyses reported here rely on the same dataset we used for analyses published in Haas, Baker, and Gonzalez (31), but here we focus on uncertainty which was not included in prior analyses. Fifty eight healthy adults (34 female and 24 male; age range: 19 to $59, M=25.4$, $S D=9.2)$ participated in the experiment. Participants were politically diverse, with 32 identifying 
as liberal and 26 identifying as conservative. Participants were recruited from the University of Nebraska-Lincoln and surrounding community. All participants were right-handed, had normal or corrected-to-normal vision, and no known history of neurological disorders. Participants were safety screened to ensure eligibility for MRI and provided informed consent in accord with study approval by the institutional review board. They were compensated $\$ 30$ USD for their participation.

\section{Experimental Design and Stimuli}

Full details of the experimental design are available in Haas, Baker, and Gonzalez (31), but the relevant details are also summarized here for convenience. We did not examine uncertainty in the prior analyses, so that variable is explained in more detail here. Participants came to the MRI center and participated in a blocked rapid event-related fMRI experiment where they evaluated the policy positions of hypothetical political candidates while undergoing MRI scanning. The experiment had a 2 uncertainty (certain/uncertain) $\times 2$ incongruence (congruent/incongruent) $\times 2$ block type (ingroup/outgroup) within-subjects design. The experimental paradigm was designed to manipulate both uncertainty and incongruence as a function of the candidates' issue positions and party identification. Prior to the start of each block, participants received information about the political candidate (Democrat or Republican) that they would be evaluating for that set of trials. All participants evaluated policy positions attributed to four different candidates (two Democrats and two Republicans) in a randomized order.

On each trial, participants received information about a specific policy position attributed to the candidate and information about both: (1) whether the candidate supports or opposes that issue and (2) the certainty with which the candidate holds that position on the issue. Specifically, participants saw one of four cues on each trial: "may support," "may oppose," "definitely supports," or "definitely opposes." Next, a policy statement appeared, and participants were asked to evaluate how they felt about the candidate's position on that issue by selecting either 
good or bad using the response pad while in the scanner $(1=$ good, $2=$ bad $)$. Each trial consisted of presentation of a cue (750 milliseconds) followed by a policy statement (4250 ms) and a jittered fixation cross (ISI: 2500, 5000, 7500, 10000, or 12500 ms). Policy statements were selected on the basis of pilot data and were selected based on clear categorization as issues that Republicans or Democrats tend to support and roughly equated on attitude extremity and importance (see 31). Participants saw each statement twice over the course of the experiment, but never the same issue twice for the same candidate. A majority of the issue positions $(66.6 \%)$ in each block were congruent with the candidate's political identification (as determined by behavioral pilot data), but a smaller subset were incongruent with his identification (33.3\% percent) to allow for examination of both congruent and incongruent issue positions. Overall, half of the trials were uncertain (50\%) and half were certain. But, uncertainty was not distributed evenly across congruent and incongruent trials. To increase external validity of the task, incongruent trials were more likely to be uncertain $(62.5 \%)$ than congruent trials (43.75\%). After the MRI portion of the study, participants completed an additional post-scan survey and responded to a series of demographic questions.

\section{MRI Data Acquisition}

MRI data were acquired using a Siemens Skyra 3.0 Tesla MRI with a 32-channel head coil. Prior to functional imaging, a high-resolution T1-weighted 3D anatomical image (MPRAGE; field of view $($ FoV $)$ read $=256 \mathrm{~mm}$, slice thickness $=1.0 \times 1.0 \times 1.0 \mathrm{~mm}$, repetition time $(T R)=$ $2400 \mathrm{~ms}$, echo time $(T E)=3.37 \mathrm{~ms}$, inversion time $(T I)=991 \mathrm{~ms}$, prescan normalize on, PAT mode $=$ GRAPPA) was collected for spatial normalization. Functional MRI data were acquired with acquisition parallel to the AC-PC line (42 slices, FoV read $=220 \mathrm{~mm}$, slice thickness $=3.0 \mathrm{x}$ $3.0 \times 3.0 \mathrm{~mm}, \mathrm{TR}=2500 \mathrm{~ms}, \mathrm{TE}=30 \mathrm{~ms}$, flip angle $=80$ degrees, prescan normalize off) . Participants completed four blocks of functional scanning, lasting approximately 8.5 minutes each. The first five volumes of each run were discarded to avoid variability due to pre-steadystate functional data. 


\section{MRI Data Preprocessing and Analysis}

MRI data were preprocessed as reported in Haas, Baker, and Gonzalez (31) using fMRI Expert Analysis Tool (FEAT) in FMRIB Software Library (FSL; 32, 33) on macOS. The highresolution 3D anatomical image (MPRAGE) was skull stripped using FSL's Brain Extraction Function (BET; 34). Data from functional runs were subjected to normalization, registration to both MPRAGE and standard space (MNI152), spatial smoothing at FWHM of $5 \mathrm{~mm}$, slice timing correction (to correct for interleaved data acquisition), and motion correction using MCFLIRT

Statistical analyses were conducted using the general linear model (GLM) as implemented in FSL. Time-series data were modeled at the first level (the trial level) using FMRIB's Improved Linear Model (FILM), and then, higher-level analysis (across sessions first, and then across subjects) was carried out using FMRIB's Local Analysis of Mixed Effects (FLAME; see 33). First, the blood oxygen level-dependent (BOLD) signal was modeled at the trial level for each run as a function of uncertainty (certain/uncertain), incongruence (congruent/incongruent), and their interaction. Data from each run were then averaged across subjects using a fixed effects model. ${ }^{1}$ The subject-level analyses were then combined into group-level region of interest (ROI) analyses using FSL FLAME1. ROI analyses on left amygdala, right amygdala, bilateral insula, and anterior cingulate cortex (ACC) were masked prior to analysis (using anatomical masks from the Harvard-Oxford Cortical/Subcortical Atlases provided with FSL) and cluster corrected for multiple comparisons. In FSL, a Z-statistic > 2.0 was used to define contiguous clusters, and then cluster probabilities were compared to the (corrected) cluster significance threshold of $p<.05$ using Gaussian random field theory (36). ${ }^{2}$

\footnotetext{
${ }^{1}$ At the subject level, we also modeled the effect of block type--whether the political candidate in each block shared the participant's political identification (ingroup candidate) or not (outgroup candidate), although this was not a primary focus of the analyses reported in this manuscript and is reported in supplemental materials.

${ }^{2}$ We also ran whole brain analyses at a more stringent threshold (cluster correction with $Z>3.0$ and $p<$ .001 ) and those results are reported in supplemental materials.
} 
In order to plot the BOLD activation to decompose interaction effects, cluster masks were created using fslmaths in FSL for each significant cluster of activation, and mean activation to each of the four conditions (certain congruent, certain incongruent, uncertain congruent, uncertain incongruent) was extracted using these cluster masks in FEATQuery. Posthoc tests were conducted in $R$, and this extracted data was used to plot MRI interaction effects (MRI summary data and syntax is available at: https://osf.io/hpv8m/).

\section{Results}

\section{Behavioral Task Data}

A within-subject ANOVA was used to examine response latency as a function of uncertainty (certain/uncertain), incongruence (congruent/incongruent), and evaluative response (good/bad). Overall, response latency was slightly faster on certain trials $(M=2567, S D=982)$ compared to uncertain trials $(M=2591, S D=961 ; F(1,51)=3.162, p=.0813)$. This effect was moderated by incongruence $(F(1,54)=4.027, p=.0498)$, such that participants were faster to respond on both certain congruent trials $(M=2542, S D=800)$ compared to certain incongruent trials $(M=2625, S D=765 ; F(1,57)=24.86, p<.001)$, as well as uncertain congruent trials $(M=$ $2567, S D=797)$ compared to uncertain incongruent trials $(M=2643, S D=801 ; F(1,57)=$ $12.72, p<.001)$ trials, but the size of the difference was a bit larger for certain relative to uncertain trials (see Figure 1). The three way interaction of uncertainty*incongruence*response was not statistically significant $(F(1,57)=1.69, p=0.199)$, but there was a significant 2-way interaction of uncertainty ${ }^{*}$ response $(F(1,50)=5.741, p=.0204)$. When participants responded good, they were faster to respond on certain trials $(M=2521, S D=819)$ relative to uncertain trials $(M=2560, S D=789 ; F(1,57)=4.268, p=.0434)$. When participants responded bad, the difference between certain trials $(M=2611, S D=782)$ and uncertain trials $(M=2624, S D=$ 
$778 ; F(1,57)=.434, p=.513)$ trials was not significant. ${ }^{3}$ Raw data and syntax for behavioral task analyses in $R$ are available at: https://osf.io/hpv8m/.

\section{fMRI Data}

Blood oxygen level-dependent (BOLD) signal was modeled as a function of uncertainty (certain/uncertain) and incongruence (congruent/incongruent). ${ }^{4} \mathrm{ROI}$ analyses revealed significant clusters of activation in our primary Regions of Interest (ROls) —insular cortex and anterior cingulate cortex (ACC)—which will be detailed below (see Table 1 for full list of significant clusters). ${ }^{5}$ Overall, we find no significant clusters of amygdala activation, which is consistent with our prior work (31).

\section{Main Effects of Uncertainty and Incongruence}

First, we examined main effects of uncertainty and incongruence by examining directional $(t)$ contrasts designed to compare differences in BOLD activation for uncertain > certain trials (and for incongruent > congruent trials). Here we examine results from ROI analyses in ACC, insula, and amygdala. These analyses revealed significant clusters of activation in bilateral insula (extending into lateral orbitofrontal cortex) and ACC that showed greater activation to certain > uncertain trials (see Figure 2). The insula analysis revealed significant clusters of activation for certain > uncertain trials on both the left (720 voxels, Z-max $=4.37, p=.000271 ;$ MNI Coordinates: $X=-32, Y=20, Z=-16)$ and right (479 voxels, Z-max $=$ 3.95, $p=.00277$ ); MNI Coordinates: $X=32, Y=28, Z=-2)$. The activation in ACC for certain > uncertain trials was centered in paracingulate gyrus (1392 voxels, Z-max $=4.14, p<.001$; MNI Coordinates: $X=-4, Y=24, Z=42$ ). Consistent with the analyses on incongruence reported in our prior work (31), we also find significant clusters of activation in right insula (409 voxels, Z-

\footnotetext{
${ }^{3}$ We also modeled the effect of block type (ingroup versus outgroup block) to examine possible interactions with uncertainty, but none of those interactions were statistically significant so we do not focus further on block type in this paper. As reported in Haas, Baker, and Gonzalez (31), we find significant main effects of incongruence and response, a significant interaction of incongruence*block type, and a marginal interaction of incongruence*response.

${ }^{4}$ Analyses including block type (ingroup versus outgroup) are reported in supplementary materials.

${ }^{5}$ Whole brain analyses are reported in supplementary materials.
} 
$\max =3.89, p=.00863 ;$ MNI Coordinates: $X=36, Y=24, Z=-2)$ and ACC (1644 voxels, Z-max $=4.15, p<.001$; MNI Coordinates: $X=-6, Y=16, Z=34)$ for incongruent $>$ congruent trials (see Figure 2). We found no significant clusters of activation in insula or ACC for the reverse contrasts (congruent > incongruent or uncertain > certain). Masked analyses for left and right amygdala showed no significant clusters of activation for any of these four directional contrasts.

\section{Interaction of Uncertainty and Incongruence}

Next, we examined the uncertainty by incongruence interaction in the same regions of interest using non-directional $(F)$ contrasts. As expected, the above main effects for uncertainty and incongruence were qualified by significant clusters of activation for the interaction in both bilateral insula and ACC (see Figure 3). As above, we find no significant clusters of activation for the interaction effect in left or right amygdala.

The larger insula cluster was centered on the left (see Figure 3a; 919 voxels, Z-max = 4.44, $p<.001$; MNI Coordinates: $X=-32, Y=24, Z=2$ ). There was also a sizeable cluster of activation centered in right insula (see Figure $3 b ; 638$ voxels, Z-max $=3.33, p=.000467 ; \mathrm{MNI}$ Coordinates: $X=42, Y=20, Z=2$ ). Decomposing the interaction effect in bilateral insula reveals a similar pattern of activation on both sides (see Figure 3). The greatest increase in activation was observed for certain incongruent trials, relative to the other three conditions, suggesting that the insula response was strongest when candidates expressed definite support for issues incongruent with their party affiliation. In left insula, activation to certain incongruent trials $(M=23.26, S D=12.50)$ was greater than for uncertain incongruent trials $(M=14.64, S D=$ 9.41; $F(1,57)=15.68, p<.001)$, but for congruent trials activation was greater for uncertain $(M$ $=18.05, \mathrm{SD}=9.35)$ than certain $(\mathrm{M}=15.62, \mathrm{SD}=7.45 ; \mathrm{F}(1,57)=2.802, \mathrm{p}=.0996)$ trials. $\operatorname{In}$ right insula, activation to certain incongruent trials $(M=32.38, S D=15.03)$ was greater than for uncertain incongruent trials $(M=20.89, S D=11.82 ; F(1,57)=19.56, p<.001)$, but for congruent trials activation was greater for uncertain $(M=23.85, S D=11.71)$ than certain $(M=$ 20.64, SD $=10.57 ; F(1,57)=2.784, p=.101)$ trials. 
In the anterior cingulate cortex (ACC), there was one large cluster of activation in response to the interaction of uncertainty and incongruence (see Figure 4). The cluster was centered on ACC / paracingulate gyrus (2548 voxels, Z-max $=4.85, p<.001$; MNI Coordinates: $X=0, Y=20, Z=42$ ). Decomposing the interaction effect in ACC shows a pattern of activation that looks similar to what was observed in bilateral insula-the greatest increase in activation occurred in response to certain incongruent trials, relative to the other three trial types. Activation to certain incongruent trials $(M=29.64, S D=10.92)$ was greater than for uncertain incongruent trials $(\mathrm{M}=21.49, \mathrm{SD}=8.59 ; \mathrm{F}(1,57)=19.73, \mathrm{p}<.001)$, but for congruent trials activation was greater for uncertain $(M=23.76, S D=9.76)$ than certain $(M=21.26, S D=7.28$; $F(1,57)=2.837, p=.0976)$ trials

\section{Discussion}

This experiment provides evidence that uncertainty impacts neural processing differently as a function of affective context. Both insula and ACC showed the greatest activation in response to policy positions that were both incongruent with candidates' party affiliation and presented with certainty. On congruent trials, the pattern was reversed but weaker, such that activation was greater to uncertain congruent versus certain congruent trials. This suggests that regions involved in evaluative processing may be especially sensitive to information that is known to be incongruent with a political candidate's stated position, perhaps so that the information can be encoded and help inform decision making later down the line.

There is an extant literature in political science on how individuals process political information and assess political candidates, yet an understanding of the psychological and neural mechanisms by which people engage in political evaluative processing is far from complete, and an understanding of the neural mechanisms underlying political evaluations is in its infancy. The primary implication of the findings presented here are that a) people attend to uncertainty in stated issue positions for political candidates and b) the effects of uncertainty on how people process political information are context-dependent. It is not uncommon that issue 
positions are incongruent with party identification, or that voters may be uncertain about where candidates truly stand on the issues, both due to lack of political knowledge and a lack of clarity from politicians. The present work suggests that evaluations of such incongruent issue positions depend on the level of certainty with which the incongruence exists. In the present work, we were agnostic as to the source of the uncertainty--participants were simply told positions were uncertain without attribution as to the cause of the uncertainty. In future work, it may be interesting to examine different types of uncertainty—for example, whether the uncertainty comes from candidates' having not made up their mind on an issue versus strategically obfuscating their position.

Relatedly, future work may want to consider variation in voters' expectations of expressed uncertainty by candidates. Voters may vary in terms of how much they expect candidates to express clarity in their issue positions. Some work suggests attitude consistency is one of the most valued qualities in a political candidate $(37,38)$, but other work in this special issue shows a nontrivial number of people just want to "watch the world burn" and may prefer some degree of uncertainty (39). Studies of individual variation in neural responses to uncertainty may improve our understanding of the electoral consequences for politicians who hold uncertain issue positions.

Future work may also want to consider voter knowledge on the issues, as it is possible those who are highly knowledgeable (and/or highly identified) may be more sensitive to these policy deviations, especially on issues with high partisan ownership (e.g., Republicans on taxes; $40,41)$. Relatedly, voters are often argued to be "issue publics" - driven predominantly by interest in a relatively small subset of issues $(42,43)$, and so another interesting avenue for future work may be to consider the role of issue importance at the individual level. Inconsistency or ambiguity on policy stances should matter more to voters who care deeply about particular issues, and those voters may even be willing to overlook inconsistencies on less important issues if a candidate has a clear stance on the issue(s) they care about most. 
This work is consistent with the view that the psychological effects of uncertainty on political cognition and behavior are context-dependent $(12,13)$-uncertainty seems to affect neural processing differently when attached to congruent versus incongruent information, at least in the neural regions examined here. This has important implications for the study of emotion and politics and political cognition more generally. Affective states such as uncertainty may sometimes be viewed as negative or attached to negative information, but can also be positively valenced. This means that we need to consider not only how individual emotions or affective states may affect processing, but how interactions of multiple affective states influence both neural processing and political behavior.

\section{Acknowledgments}

This work was supported by the University of Nebraska-Lincoln Office for Research and Economic Development, College of Arts and Sciences, Center for Brain, Biology, and Behavior, and Department of Political Science. The UNL Political Attitudes and Cognition Lab and MRI Users Group provided useful feedback on this work. Additional thanks to undergraduate research assistants Allison Haindfield, Grace Stallworth, and Sarah Sweeney and MRI Technologist Joanne Murray for assistance with data collection. 


\section{References}

1. Downs A. An economic theory of political action in a democracy. Journal of Political Economy. 1957;65:135-50.

2. Alvarez RM, Franklin C. Uncertainty and political perceptions. Journal of Politics. 1994;56:671-88.

3. McGraw KM, Hasecke E, Conger K. Ambivalence, uncertainty, and processes of candidate evaluation. Political Psychology. 2003;24:421-48.

4. Fearon JD. Domestic political audiences and the escalation of international disputes. The American Political Science Review. 1994;88:577-92.

5. Tomz M. Domestic Audience Costs in International Relations: An Experimental Approach. International Organization. 2007;61(04).

6. Croco SE. The flipside of flip-flopping: Leader inconsistency, citizen preferences, and the War in Iraq. Foreign Policy Analysis. 2016;12(3):237-57.

7. McDonald J, Croco SE, Turitto C. Teflon Don or Politics as Usual? An Examination of Foreign Policy Flip-Flops in the Age of Trump. The Journal of Politics. 2019;81(2):757-66.

8. Tomz M, Van Houweling RP. The Electoral Implications of Candidate Ambiguity. American Political Science Review. 2009;103(01):83.

9. MacKuen M, Marcus GE, Neuman WR, Keele L. The third way: The theory of affective intelligence and American democracy. In: Neuman WR, Marcus GE, Crigler AN, MacKuen M, editors. The affect effect: Dynamics of emotion in political thinking and behavior. Chicago: University of Chicago Press; 2007. p. 124-51.

10. MacKuen M, Wolak J, Keele L, Marcus GE. Civic engagements: Resolute partisanship or reflective deliberation. American Journal of Political Science. 2010;54:440-58.

11. Marcus GE, Neuman WR, MacKuen M. Affective intelligence and political judgment. Chicago: University of Chicago Press; 2000. 
12. Haas IJ, Cunningham WA. The uncertainty paradox: Perceived threat moderates the effect of uncertainty on political tolerance. Political Psychology. 2014;35(2):291-302.

13. Haas IJ. The impact of uncertainty, threat, and political identity on support for political compromise. Basic and Applied Social Psychology. 2016;38(3):137-52.

14. Cunningham WA, Haas IJ, Jahn A. Attitudes. In: Decety J, Cacioppo JT, editors. The Oxford Handbook of Social Neuroscience. New York, NY: Oxford University Press; 2011. p. 212-26.

15. Cunningham WA, Zelazo PD. Attitudes and evaluations: A social cognitive neuroscience perspective. Trends in Cognitive Sciences. 2007;11:97-104.

16. Cunningham WA, Zelazo PD, Packer DJ, Van Bavel JJ. The iterative reprocessing model: A multilevel framework for attitudes and evaluation. Social Cognition. 2007;25:736-60. 17. Kaplan JT, Freedman J, lacoboni M. Us versus them: Political attitudes and party affiliation influence neural responses to faces of presidential candidates. Neuropsychologia. $2007 ; 45: 55-64$.

18. Spezio ML, Rangel A, Alvarez RM, O'Doherty JP, Mattes K, Todorov A, et al. A neural basis for the effect of candidate appearance on election outcomes. Social Cognitive and Affective Neuroscience. 2008;3(4):344-52.

19. Tusche A, Kahnt T, Wisniewski D, Haynes JD. Automatic processing of political preferences in the human brain. Neuroimage. 2013;72:174-82.

20. Kolling N, Behrens T, Wittmann MK, Rushworth M. Multiple signals in anterior cingulate cortex. Current Opinion in Neurobiology. 2016;37:36-43.

21. Botvinick MM. Conflict monitoring and decision making: Reconciling two perspectives on anterior cingulate function. Cognitive, Affective, \& Behavioral Neuroscience. 2007;7(4):356-66. 22. Carter CS, Braver TS, Barch DM, Botvinick MM, Noll D, Cohen JD. Anterior cingulate cortex, error detection, and the online monitoring of performance. Science. 1998;280:747-9. 
23. Rushworth MF, Behrens TE. Choice, uncertainty and value in prefrontal and cingulate cortex. Nat Neurosci. 2008;11(4):389-97.

24. Yu R, Zhou W, Zhou X. Rapid processing of both reward probability and reward uncertainty in the human anterior cingulate cortex. PLoS ONE. 2011;6:e29633.

25. Harris S, Sheth SA, Cohen MS. Functional neuroimaging of belief, disbelief, and uncertainty. Annals of neurology. 2008;63(2):141-7.

26. Uddin LQ. Salience processing and insular cortical function and dysfunction. Nature Reviews Neuroscience. 2015;16(1):55-61.

27. Gu X, Liu X, Van Dam NT, Hof PR, Fan J. Cognition-emotion integration in the anterior insular cortex. Cerebral Cortex. 2013;23(1):20-7.

28. Singer T, Critchley HD, Preuschoff K. A common role of insula in feelings, empathy and uncertainty. Trends in Cognitive Sciences. 2009;13(8):334-40.

29. Grinband J, Hirsch J, Ferrera VP. A neural representation of categorization uncertainty in the human brain. Neuron. 2006;49(5):757-63.

30. Medford N, Critchley HD. Conjoint activity of anterior insular and anterior cingulate cortex: awareness and response. Brain Structure \& Function. 2010;214(5-6):535-49.

31. Haas IJ, Baker MN, Gonzalez FJ. Who can deviate from the party line? Political ideology moderates evaluation of incongruent policy positions in insula and anterior cingulate cortex. Social Justice Research. 2017;30(4):355-80.

32. Jenkinson M, Beckmann CF, Behrens TE, Woolrich MW, Smith SM. FSL. Neurolmage. 2012;62:782-90.

33. Smith SM, Jaenkinson M, Woolrich Mw, Beckmann CF, Behrens TEJ, Johansen-Berg H, et al. Advances in functional and structural MR image analysis and implementation as FSL. Neurolmage. 2004;23:S208-S19.

34. Smith SM. Fast robust automated brain extraction. Human brain mapping. 2002;17:14355. 
35. Jenkinson M, Bannister P, Brady M, Smith S. Improved optimisation for the robust and accurate linear registration and motion correction of brain images. Neurolmage. 2002;17:82541.

36. Worsley KJ. Statistical analysis of activation images. In: Jezzard P, Matthews PM, Smith SM, editors. Functional MRI: An Introduction to Methods: Oxford University Press; 2001. 37. Kinder DR, Peters MD, Abelson RP, Fiske ST. Presidential prototypes. Political Behavior. 1980;2(4):315-37.

38. McGraw KM, Fischle M, Stenner K, Lodge M. What's in a word? Political Behavior. 1996;18(3):263-87.

39. Arceneaux K, Gravelle TB, Osmundsen M, Peterson MB, Reifler J, Scotto TJ. Some people just want to watch the world burn: The prevalence, psychology and politics of the "Need for Chaos". Philosophical Transactions of the Royal Society B.

40. Budge I, Farlie D. Explaining and Predicting Elections: Issue Effects and Party Strategies in Twenty-Three Democracies. London: George Allen \& Unwin; 1983.

41. Stokes DE. Spatial models of party competition. The American Political Science Review. 1963;57(2):368-77.

42. Converse PE. The nature of belief systems in mass publics. In: Apter DE, editor. Ideology and discontent. New York: Free Press; 1964. p. 206-61.

43. Krosnick JA. Government policy and citizen passion: A study of issue publics in contemporary America. Political Behavior. 1990;12:59-92. 


\section{Figure Captions}

Figure 1. Raw response latency (in milliseconds) as a function of uncertainty (certain/uncertain) and incongruence (congruent/incongruent).

Figure 2. BOLD activation in (2a) ACC and (2b) bilateral insula in response to certain > uncertain trials (red-yellow) and incongruent > congruent trials (blue-light blue). Images were created by overlaying the thresholded Z-statistic images on a standard space template (MNI152) and are centered on the peak voxel for (2a) the certain > uncertain contrast in ACC and $(2 b)$ the certain $>$ uncertain contrast in right insula from the ROI analyses.

Figure 3. BOLD activation in bilateral insula in response to the interaction of uncertainty and incongruence. Images are centered on peak voxel for each cluster from the ROI analysis: (a) left insula (MNI Coordinates: $X=-32, Y=24, Z=2$ ) and $(b)$ right insula (MNI Coordinates: $X=$ 42, $Y=20, Z=2$ ). Plots represent mean parameter estimates for each of the four trial types (certain congruent, uncertain congruent, certain incongruent, uncertain incongruent), extracted from functional cluster masks and separated by cluster. Error bars on the bar graphs represent within-subject standard errors.

Figure 4. BOLD activation in ACC in response to the interaction of uncertainty and incongruence. Images are centered on peak voxel of activation from the ROI analysis (MNI Coordinates: $X=0, Y=20, Z=42$ ). Plots represent mean parameter estimates for each of the four trial types (certain congruent, uncertain congruent, certain incongruent, uncertain incongruent), extracted from the functional cluster mask. Error bars on the bar graphs represent within-subject standard errors. 
Figure 1. Raw response latency (in milliseconds) as a function of uncertainty (certain/uncertain) and incongruence (congruent/incongruent).

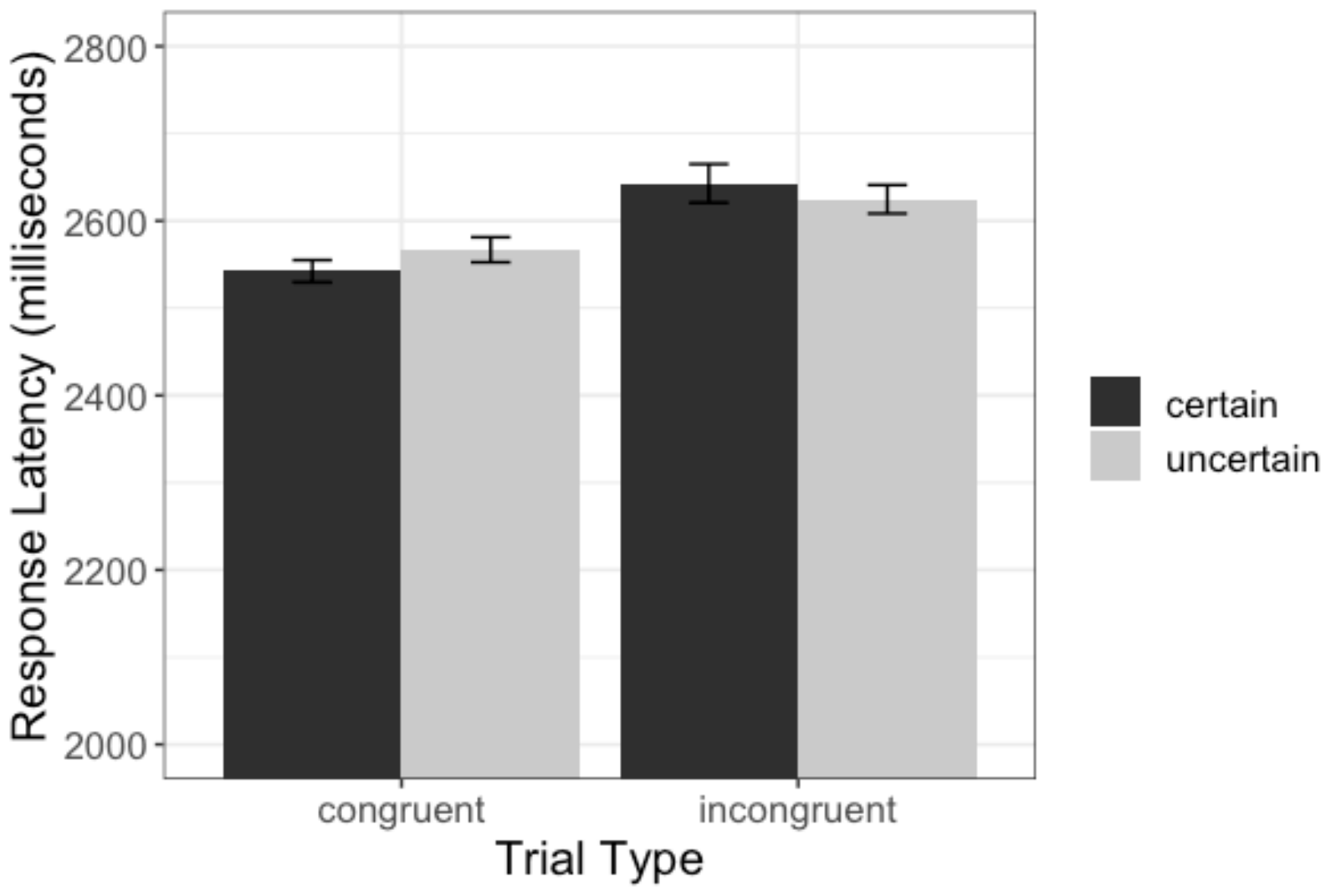


Figure 2. BOLD activation in (2a) ACC and (2b) bilateral insula in response to certain > uncertain trials (red-yellow) and incongruent > congruent trials (blue-light blue). Images were created by overlaying the thresholded Z-statistic images on a standard space template (MNI152) and are centered on the peak voxel for $(2 a)$ the certain $>$ uncertain contrast in ACC and $(2 b)$ the certain $>$ uncertain contrast in right insula from the ROI analyses.

(2a) ACC

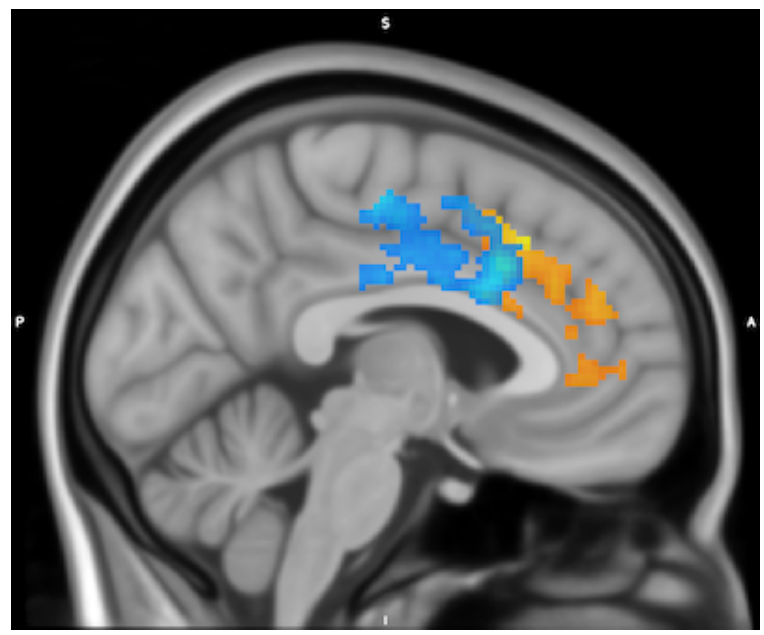

(2b) bilateral insula

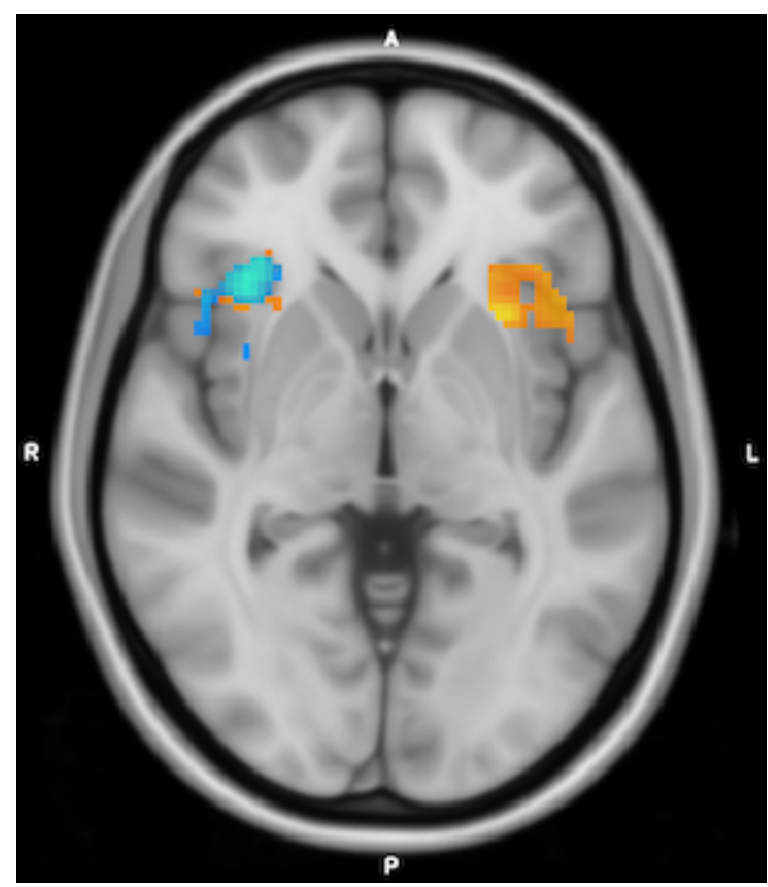


Figure 3. BOLD activation in bilateral insula in response to the interaction of uncertainty and incongruence. Images are centered on peak voxel for each cluster from the ROI analysis: (a) left insula (MNI Coordinates: $X=-32, Y=24, Z=2$ ) and (b) right insula (MNI Coordinates: $X=$ 42, $Y=20, Z=2$ ). Plots represent mean parameter estimates for each of the four trial types (certain congruent, uncertain congruent, certain incongruent, uncertain incongruent), extracted from functional cluster masks and separated by cluster. Error bars on the bar graphs represent within-subject standard errors.

(3a) left insula

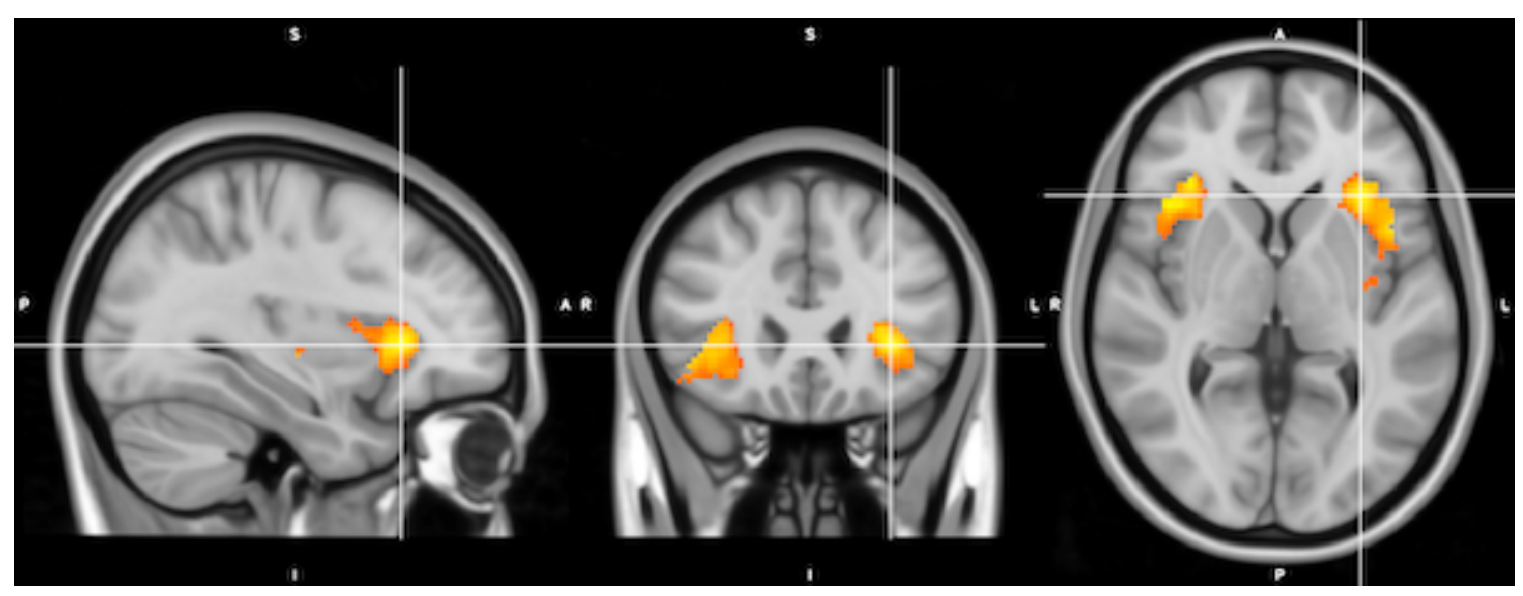

\section{Left Insula}

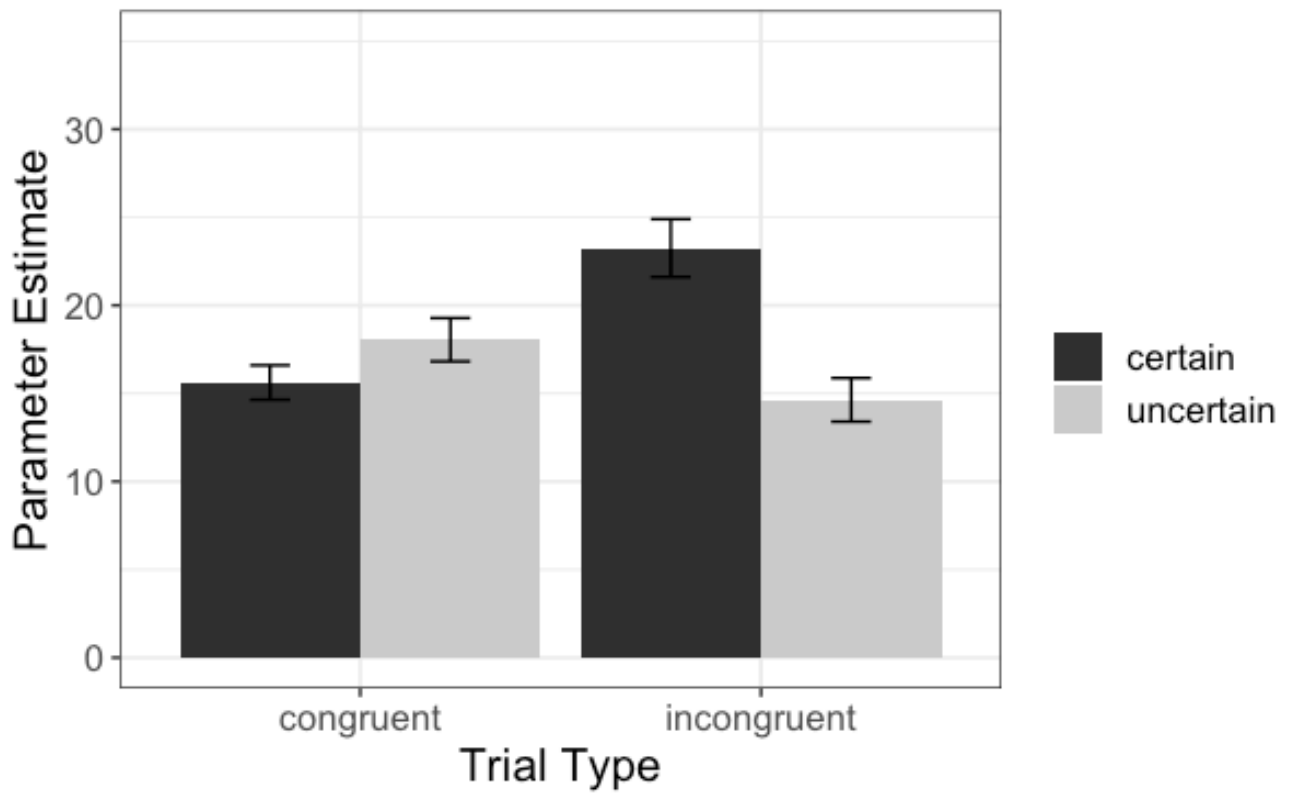


(3b) right insula

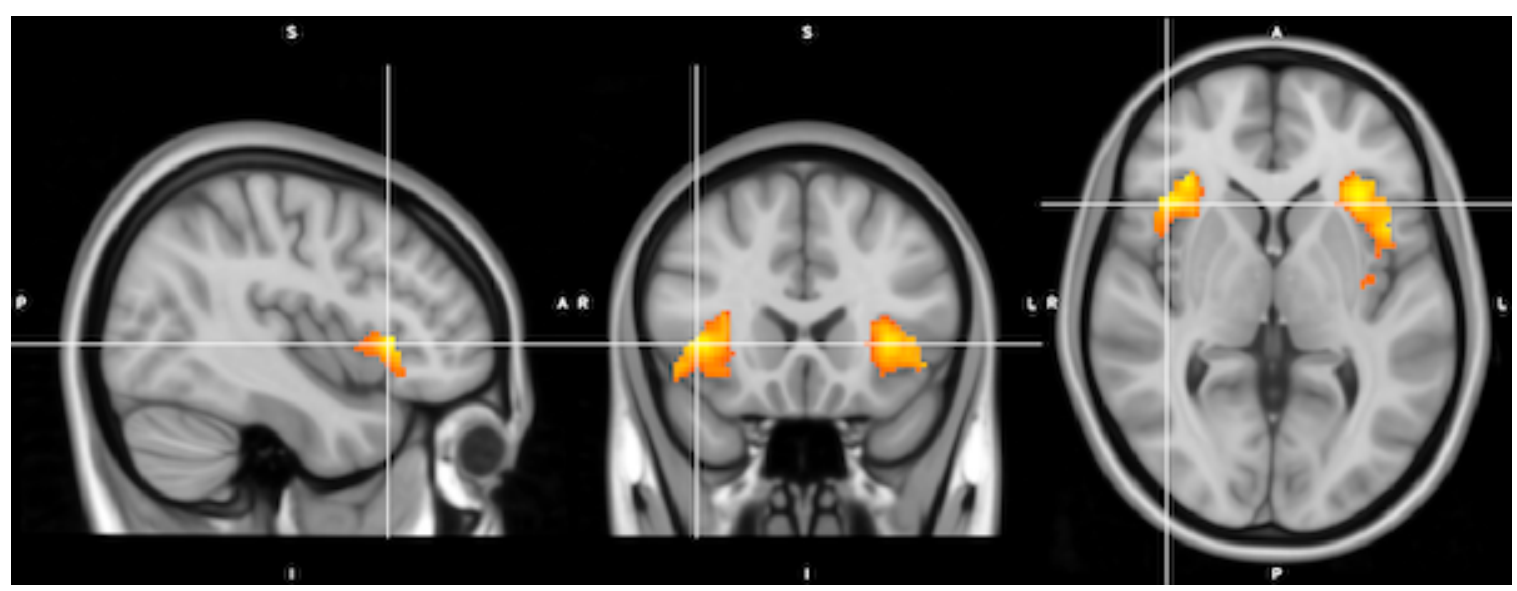

Right Insula

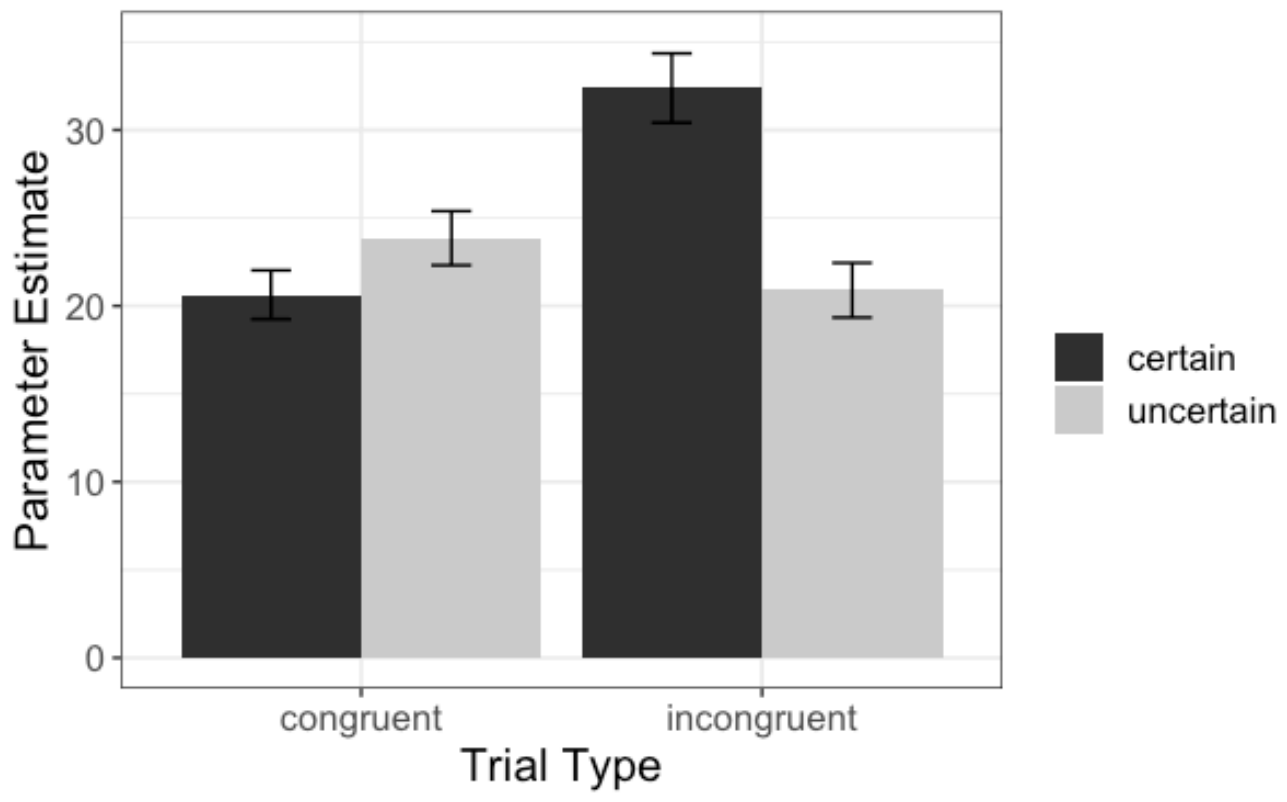


Figure 4. BOLD activation in ACC in response to the interaction of uncertainty and incongruence. Images are centered on peak voxel of activation from the ROI analysis (MNI Coordinates: $X=0, Y=20, Z=42$ ). Plots represent mean parameter estimates for each of the four trial types (certain congruent, uncertain congruent, certain incongruent, uncertain incongruent), extracted from the functional cluster mask. Error bars on the bar graphs represent within-subject standard errors.

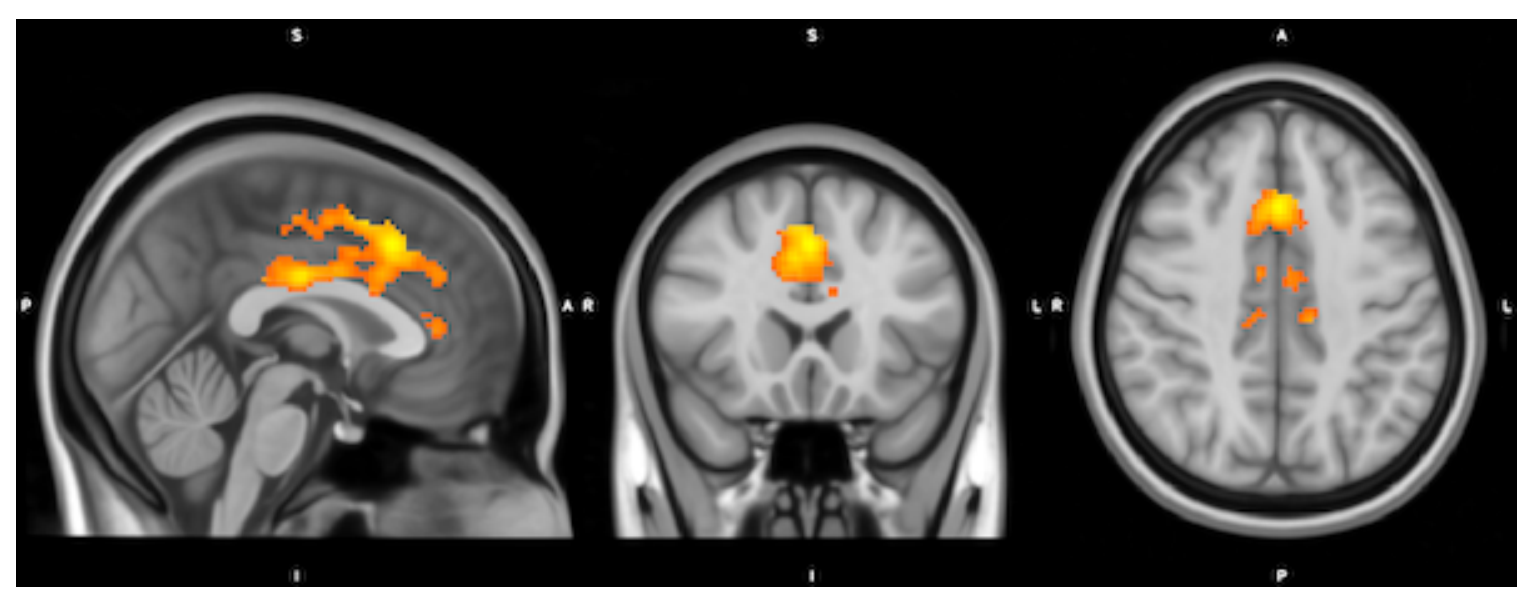

Anterior Cingulate Cortex (ACC)

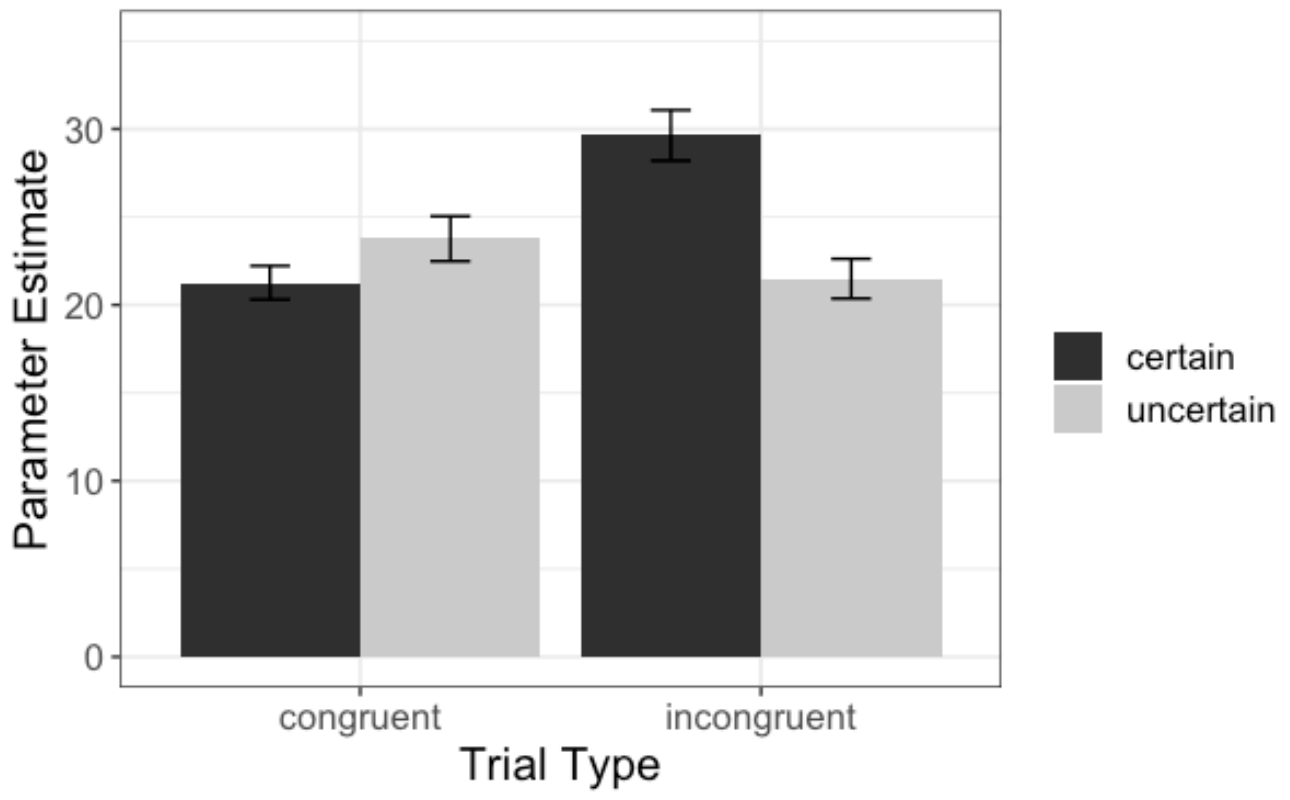


Table 1. Significant clusters of BOLD activation in insula and ACC for main effects and interactions of uncertainty (certain/uncertain) and incongruence (congruent/incongruent). X, Y, Z coordinates are in $\mathrm{MNI}$ space. Cluster information for main effects is based on directional contrasts ( $t$ tests) and cluster information for interactions is based on non-directional contrasts ( $F$-tests).

\begin{tabular}{|c|c|c|c|c|c|c|c|c|}
\hline Contrast & $\begin{array}{l}\text { Anatomical } \\
\text { Label(s) }\end{array}$ & Side & $\begin{array}{l}\text { Cluster } \\
\text { size }\end{array}$ & $p$ value & $\begin{array}{l}\text { Peak } \\
\text { activation } \\
\text { (Z score) }\end{array}$ & $X$ & $\mathbf{Y}$ & $\mathbf{Z}$ \\
\hline Certain > Uncertain & $\begin{array}{l}\text { Insular cortex; } \\
\text { lateral orbitofrontal } \\
\text { cortex }\end{array}$ & left & 720 & .000271 & 4.37 & -32 & 20 & -16 \\
\hline Certain > Uncertain & $\begin{array}{l}\text { Insular cortex; } \\
\text { lateral orbitofrontal } \\
\text { cortex }\end{array}$ & right & 479 & .00277 & 3.95 & 32 & 28 & -2 \\
\hline Certain > Uncertain & paracingulate gyrus & - & 1392 & $<.001$ & 4.14 & -4 & 24 & 42 \\
\hline Incongruent > Congruent & $\begin{array}{l}\text { Insular cortex; } \\
\text { lateral orbitofrontal } \\
\text { cortex }\end{array}$ & right & 409 & .00863 & 3.89 & 36 & 24 & -2 \\
\hline Incongruent > Congruent & $\begin{array}{l}\text { cingulate gyrus, } \\
\text { anterior division; } \\
\text { paracingulate gyrus }\end{array}$ & - & 1644 & $<.001$ & 4.15 & -6 & 16 & 34 \\
\hline Uncertainty $\mathrm{x}$ Incongruence & $\begin{array}{l}\text { Insular cortex; } \\
\text { lateral orbitofrontal } \\
\text { cortex }\end{array}$ & left & 919 & $<.001$ & 4.44 & -32 & 24 & 2 \\
\hline Uncertainty $\mathrm{x}$ Incongruence & $\begin{array}{l}\text { Insular cortex; } \\
\text { lateral orbitofrontal } \\
\text { cortex }\end{array}$ & right & 638 & .000467 & 3.33 & 42 & 20 & 2 \\
\hline Uncertainty $\mathrm{x}$ Incongruence & $\begin{array}{l}\text { Paracingulate } \\
\text { gyrus; cingulate } \\
\text { gyrus, anterior } \\
\text { division }\end{array}$ & - & 2548 & $<.001$ & 4.85 & 0 & 20 & 42 \\
\hline
\end{tabular}

